\title{
Assessing relative contributions of PAHs to soot mass by reversible heterogeneous nucleation and condensation
}

\author{
Nick A. Eaves ${ }^{\mathrm{a}}$, Seth B. Dworkin ${ }^{\mathrm{b}}$, Murray J. Thomson,* \\ ${ }^{a}$ Department of Mechanical and Industrial Engineering, University of Toronto, 5 Kings \\ College Road, Toronto, Ontario, Canada, M5S $3 G 8$ \\ ${ }^{b}$ Department of Mechanical and Industrial Engineering, Ryerson University, 350 \\ Victoria St, Toronto, Ontario, Canada, M5B $2 K 3$
}

\section{Abstract}

Given the recent EURO 6 regulations, which include limits on particle number density (and hence size) for soot emissions from land vehicles, soot models must be capable of accurately predicting soot particle sizes. Previous modeling work has demonstrated the importance of the relative strengths of nucleation and condensation in predicting soot primary particle size. Due to this importance, a fundamental reversible model for nucleation and condensation, called the reversible PAH clustering (RPC) model, was developed in previous work through the use of statistical mechanics and the results from several recent works.

In the present work, the RPC model is enhanced to include multiple nucleation (or dimerization) events from 6 different PAH size groups, resulting in 21 unique dimer pairs. In addition, a soot PAH tracking model is developed to track the amount of each PAH size group within soot particles. The addition of this model resulted in reduced computation times and the ability

\footnotetext{
*Corresponding author. Fax $+1 \quad 416 \quad 978$ 7753. Email Address:murray.thomson@utoronto.ca (M.J. Thomson).
} 
to investigate PAH-PAH reactions within soot particles. The results of the enhanced RPC model demonstrate that smaller PAHs are most important for the nucleation process, while small and large PAHs are important for the condensation process. These results are shown to be due to the relatively lower reversibility of condensation versus the nucleation process. These findings are discussed in light of recent experimental results in the literature and are shown to be well supported.

Keywords: reversibility, PAH nucleation, PAH condensation, laminar diffusion flame, soot model

\section{Introduction}

Reducing soot emissions from combustion processes is important due to the negative health and environmental effects of atmospheric soot. Due to the highly complex nature of soot formation, detailed numerical models are employed to gain fundamental understanding of the factors that affect each mechanism of soot evolution. The recent European emission standard for land vehicles, EURO 6, includes regulations on particle number density in addition to total particle mass (and hence particle size), thus accurate modeling of particle number density and size is important. In order to accurately predict soot particle size for a wide range of conditions, numerical models must be able to accurately model nucleation and condensation, and their relative contributions to total soot mass, without reliance on tunable efficiencies for these processes. 


\subsection{PAH clustering during soot formation}

There has been a significant amount of recent investigations into the nature of physical PAH nucleation and condensation. In work by Sabbah et al. [1], it was shown that at flame temperatures, the dimerization of two pyrene molecules was not thermodynamically favored. It was asserted in that work that any proposed efficiency for the nucleation process should be a function of equilibrium ratios or constants. There has been some work into attempting to define an equilibrium constant for the dimerization of other PAHs as well. In a review article by Wang [2], statistical mechanics was utilized to define an equilibrium constant for the dimerization of pyrene, ovalene, and circumcoronene using binding energies taken from [3]. Assumptions had to be made regarding the average frequency of the vibration modes created during the dimerization process. Investigations have been performed into the magnitude of these vibration modes in the work of Totton et al. [4] for a range of dimers. In Totton et al. [4], a more advanced model for determining the binding energies between PAHs was developed. Rapacioli et al. [5, 6] looked at the frequencies of the vibration modes for coronene stacks containing up to 8 coronenes and vibration modes for various PAH dimers. Their conclusion was that in general, as PAH stack sizes increase, as would occur during the condensation process, vibration frequencies reduced. In Elvati and Violi [7], and Chung and Violi [8], it was determined that the addition of aliphatic chains, and not only a PAH's size, influence whether or not a dimerization was favored. Finally, Lowe and Violi [9] expanded on the work by investigating heterogeneous dimer pair stability. Their work suggested that the reduced mass of the pair was the main factor in determining dimer 
stability. All of these works reached the conclusion that PAHs much larger than pyrene (ovalene and larger) would need to be present for physical PAH attraction to play a role in nucleation and condensation.

The experimental work performed by Teini et al. [10], in which High Resolution Transmission Electron Microscopy (HRTEM) was used to investigate the types of particles found within nascent soot, conflicted with the results of these recent thermodynamic investigations. The HRTEM showed that the sizes of particles within nascent soot were fairly constant at the core and edges, and consistent with PAHs containing approximately 20 carbon atoms, or 5-rings. This finding is consistent with the much earlier work of [11], where laser micro-probe mass spectrometry (LMMS) was used to determine the mass of PAHs found within nascent soot particles. The masses were found to be consistent with PAHs consisting of approximately 20 to 21 carbons. It should be noted that in [11], PAHs containing as many as 38 carbons where detected in more mature soot particles. To reconcile the experimental observations with the theoretical works, it has been suggested that PAH-based nucleation and condensation may not be controlled by equilibrium, but rather the overall kinetics (forward and reverse rate), for which accounting for reversibility is highly important.

Given this evidence, in Eaves et al. [12], the present authors asserted that the nucleation and condensation processes should be modeled as reversible events. A novel, fundamental, reversible PAH clustering (RPC) model was developed which utilized the recent studies into physical PAH stacking and statistical mechanics to derive the reverse rates of nucleation and condensation. It was demonstrated that when considering nucleation as 
a reversible event, condensation should be considered reversible (as opposed to being efficiency-based) as well to give best agreement with experimental data. While the development of this model was a significant advancement, there were three limitations of the model as originally conceived. The model only considered one homogeneous dimerization event (of benzo-a-pyrene, or A5-A5), assumed that once a cluster larger than two PAHs formed that it rapidly became amorphous, mature soot, and was very computationally intensive.

\subsection{Agenda}

In this present work, the RPC model is expanded to include nucleation and condensation from a wide range of PAH size groups. The surface PAH tracking model is replaced with a total soot PAH tracking model to allow for less computational effort and provide the ability to account for PAH reactions, or carbonization, within soot particles. The performance of the new model is assessed and is utilized to elucidate the key PAH contributors to the nucleation and condensation processes.

\section{Problem formulation}

The flame chosen for this investigation is the steady, non-smoking, co-

flow laminar diffusion ethylene-air flame originally investigated in [13], and since exhaustively studied by multiple groups. This flame has published experimental data for soot aggregate and primary particle size and number densities. These characteristics make it an excellent choice for comparisons of various soot models. This flame has been extensively studied both exper- 
imentally and numerically, thus the details of the burner and flame may be viewed in $[14-16]$.

For the gaseous phase, the fully coupled elliptical conservation equations for mass, momentum, energy, and species mass fraction are solved. CoFlame [17], a parallelized sooting laminar flame solver, utilizes the axi-symmetrical nature of the flame, and equations are solved in the two-dimensional ( $z$ and r) cylindrical co-ordinate system. Conjugate heat transfer between the solid fuel tube and the fuel and air streams is modeled using the harmonic mean method $[18,19]$. The chemical kinetic mechanism that is used for this investigation is the one originally presented in [20], with modifications described in $[21,22]$. Soot particle dynamics are described using a fixed sectional method, in which soot particle mass ranges are divided logarithmically into discrete sections. The soot sectional model includes several processes, those being nucleation, PAH condensation, HACA surface growth, surface oxidation, coagulation, fragmentation, particle diffusion, and thermophoresis.

Nucleation and condensation are modeled as the physical binding of PAHs to other PAHs (nucleation), or clusters of PAHs or soot particles (condensation). The nucleation and condensation model employed is referred to as the reversible PAH clustering (RPC) model. The nucleation process is treated as fully reversible, while the condensation process is treated reversibly although net negative condensation rates are disallowed to maintain numerical stability. Statistical mechanics is utilized to determine the enthalpy and entropy of the nucleation and condensation processes for any arbitrary PAH-PAH or PAH-PAH cluster or soot collision event. These values can in turn be used to determine an equilibrium constant $[2,4]$ and subsequently reverse 
rate coefficients [12]. Further description of the RPC Model is available in the Supplemental Material. All other soot processes, including nucleation, surface growth, PAH surface condensation, surface oxidation, coagulation, fragmentation, particle diffusion, thermophoresis, and particle radiation are modeled in the same fashion as in [12, 19, 21, 23-33]. A detailed description of the governing equations, boundary conditions, solution methodology, and chemical mechanism can be found in previous works [17].

\section{Model development}

The RPC model, originally proposed in [12], is modified to allow nucleation or condensation to occur from a wide range of PAH size groups. The RPC model is expanded to include soot nucleation and condensation from the dimerization of 6 different PAH size groups, those being PAHs containing 10 (A2),12 (A2R5, P2), 14 (A3), 16 (A4), 18 (BGHIF, A4C2H), or 20 (BAPYR, the largest PAH in the mechanism) carbons (representative PAHs are in parenthesis). Multiple homogeneous and heterogeneous dimer pairs are tracked. The equations utilized to track surface PAHs are replaced by those for tracking PAHs contained within soot particles so that the effects of carbonization can be investigated and possible reductions in computation time. Carbonization is modeled as a process of internal PAHs within the soot particles bonding with each other, leading to a more layered, graphitic, structure. In total, the enhanced RPC model solves 322 equations at each control volume to resolve soot morphology and composition, with 21 dimer sections

containing 2 equations per section (chemically and non-chemically bonded dimers), and 35 aggregate sections with 7 equations per section (primary 
particles, and 6 groups of internal PAHs).

\subsection{PAH dimers}

Given the 6 different size groups, there are 21 unique combinations possible for a dimerization, or nucleation, event. To accurately track each combination, the first 21 sections of the particle dynamics model are treated as dimer sections. In these sections, there are only two equations per section, those being the number density of aggregates and the number density of dimers, as the primary particle and PAH number densities of these sections are prescribed. The equation for the number density of aggregates for these dimer sections are the same as originally described in [32] for the first section. The number density of dimers equation is similar; however, an additional source term from the effect of carbonization is included. By including the effect of carbonization, the rate at which physically bonded $\mathrm{PAH}$ dimers reverse into PAH monomers is reduced as the carbonization rate will reduce the number density of dimers. The difference between the number density of aggregates and number density of dimers in the first 21 sections would represent PAH dimers that are chemically, rather than physically, bonded. For a list of all dimer pairs, the reader is referred to the Supplemental Material.

The binding energies for the various dimer pairs are calculated based on the binding energy for pyrene from Totton et al. [4] and the linear scaling of binding energy with collisional reduced mass developed by Herdman and Miller [3]. The frequencies of the six vibration modes that are created during a dimerization event are taken from [12] and are kept the same for all possible dimerization events. It is shown in previous works that the average vibration frequency does not change significantly between different homogenous 
dimers [12]. While there are very limited studies on heterogeneous dimerization, Lowe et al. [9] did demonstrate that the difference in free energies of dimerization for heterogeneous pairs are primarily due to alterations in binding energies, rather than entropic, or vibrational frequency, effects. Thus, the assumption that the average vibration frequency for heterogeneous dimers is constant and equal to that of homogeneous dimers is applied.

\subsection{Soot PAH number density}

In order to utilize the RPC model, there must be information about the PAH content of the soot particles in each section such that the reverse condensation rates can be determined. Therefore, an additional conservation equation is solved per section for the PAH number density for each $\mathrm{PAH}$ group included in the condensation process in a similar fashion as primary particles are tracked in each section $[34,35]$. One additional term in the soot $\mathrm{PAH}$ number density equation is the carbonization rate, which is given by:

$$
\left.\frac{\partial N_{i}^{p a h}}{\partial t}\right|_{c a}=A \exp \left(\frac{E_{A}}{R_{u} T}\right)
$$

where $\mathrm{A}$ is the pre-exponential term, and $E_{A}$ is the activation energy. The value of $E_{A}$ is taken from $[11,36]$. The value of $A$ in the literature varies by six orders of magnitude; however, in this study it is set to $1.78 * 10^{10}$, which is in the range of the values reported in literature $[11,36,37]$ and provides the best agreement with experimental data. It should be noted that without modeling the carbonization process, soot volume fractions are under-predicted by several orders of magnitude. This is consistent with all recent studies on PAH clustering processes, which demonstrate that physical 
bonding alone cannot be the sole mechanism of soot formation and that chemical processes must be involved [4, 9, 38]. A detailed study of the effect of varying the carbonization rates on predicted soot morphology is left for future work. For the conservation equation and the other source terms in the soot PAH number density equation, the reader is referred to the Supplemental Material.

\section{Results and discussion}

\subsection{Model performance}

\subsubsection{Computational cost}

The original RPC model, starting from an initial guess containing an accurate temperature field and major species concentrations, takes approximately 420000 CPU-Hours utilizing 384 Intel Xeon processors to reach a converged solution for the co-flow laminar diffusion ethylene-air flame originally investigated in [13]. The original RPC model only considered two PAH size groups and 140 soot transport equations. For the same flame, computational domain, and number of processors, the enhanced RPC model takes approximately 101000 CPU-Hours, while considering 6 PAH size groups and solving 322 soot transport equations. Since computational time is approximately proportional to the number of equations to the power of 2 for the CoFlame code, the enhanced RPC model computationally costs approximately 20 times less than the original RPC model for an equal number of conservation equations. This reduction in cost is attributed to tracking the total PAH number density within soot particles, rather than attempting to track soot surface PAH number density, which was numerically cumbersome. 


\subsubsection{Soot morphology predictions}

The performance of the enhanced RPC model is assessed based on comparisons to data available for the co-flow laminar diffusion ethylene-air flame originally investigated in [13] and results for the original RPC model [12].
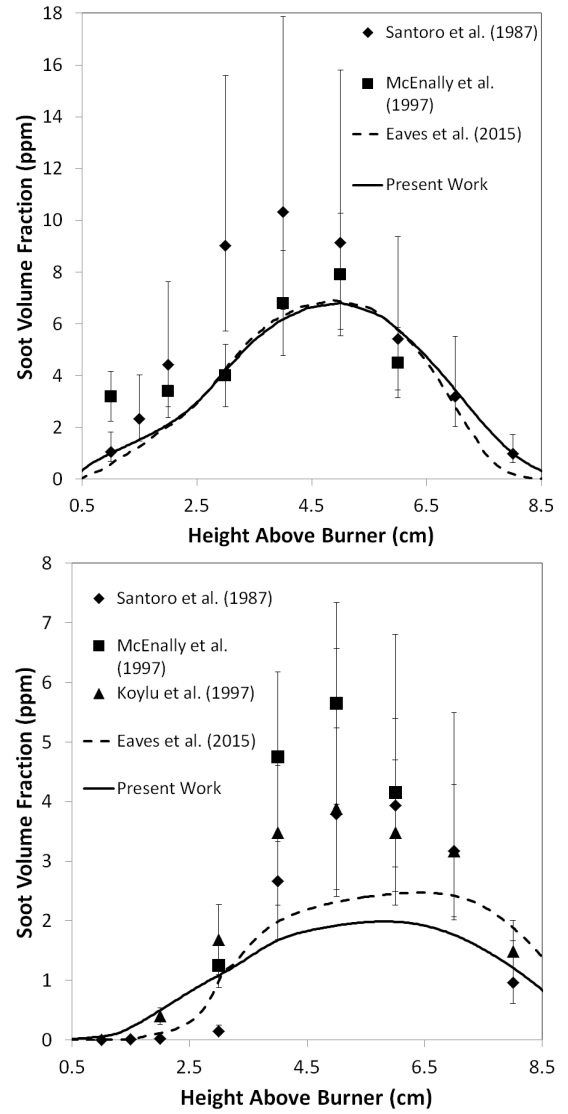

Figure 1: Comparison of experimental values and those computed with the CoFlame code for soot volume fraction along the pathline of maximum soot on the wings (top) and the centerline (bottom) for the ethylene-air Santoro Flame [13, 39, 40].

Fig. 1 displays a comparison between the experimental values and those computed with the CoFlame code with the original and enhanced RPC model for soot volume fraction along the pathline of maximum soot along the wings 
(top), and the centerline (bottom). Both models produce very similar results along the wings and centerline of the flame. The predictions are within the experimental uncertainty along the wings, although there are under predictions of the peak soot volume fraction along the centerline. Numerous studies have displayed that wing soot formation in this particular flame, and other diffusion flames, is dominated by acetylene addition; however, recent studies suggest other species may be important as well [41, 42].

Fig. 2 displays a comparison of $N_{a}$ (aggregate number density) along the pathline of maximum soot along the wings (left), and the centerline (right). While the original RPC model predictions are not within experimental uncertainty, the enhanced RPC model is within uncertainty for the majority of axial height locations along the wings and centerline. The enhanced RPC model provides improved predictions for aggregate number density at lower axial heights. This improved prediction is discussed in section 4.2.
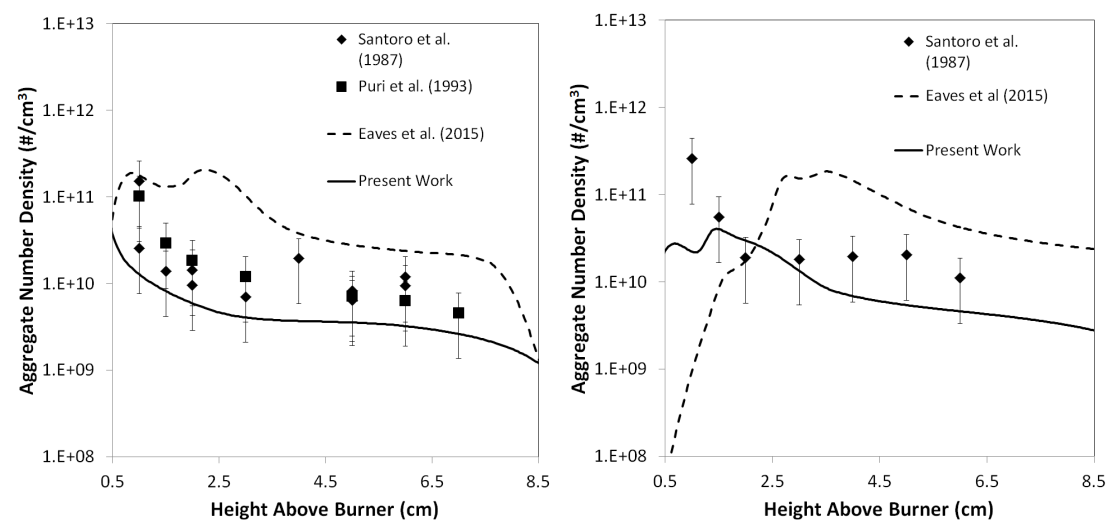

Figure 2: Comparison of experimental values and those computed with the CoFlame code for aggregate number density $\left(N_{a}\right)$ along the pathline of maximum soot along the wings (left), and the centerline (right) for the ethylene-air Santoro Flame [14, 16].

The reader is referred to the Supplementary Material for comparisons 
with other morphological parameters (number density of primaries, primary particle size, and number of primaries per aggregate).

\subsection{Contribution of each PAH size group to nucleation and condensation}

Due to the uncertainties associated with the carbonization rate, the contribution of each PAH to nucleation and condensation is investigated for four different carbonization rates with different orders of magnitude for the preexponential term. Model C11 has a pre-exponential of $1.78 \times 10^{11}$, Model C10 $1.78 \times 10^{10}$ and so on.
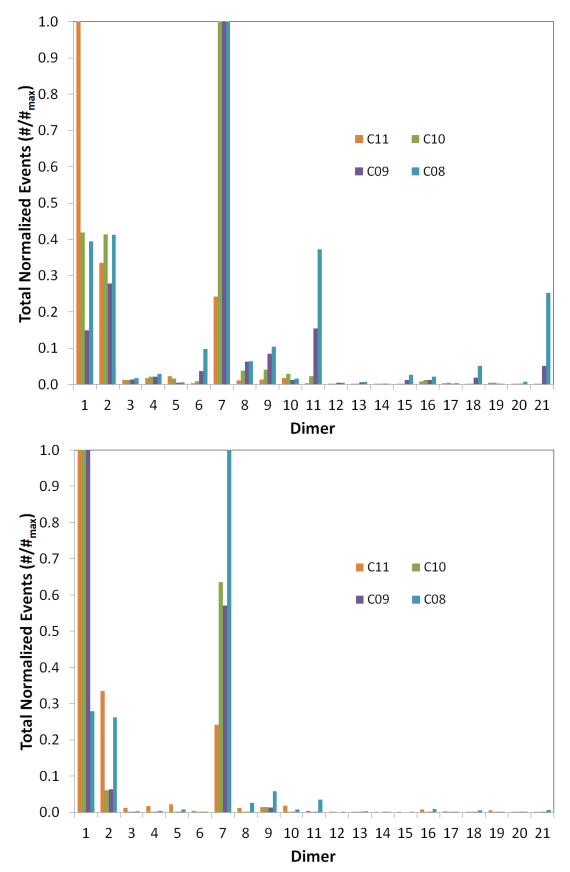

Figure 3: Total path integrated contribution to soot nucleation for each of the 21 dimer pairs for four models along the pathline of maximum soot along the wings (top), and the centerline (bottom) for a co-flow ethylene-air diffusion flame.

Fig. 3 displays the total path integrated contribution to soot nucleation for each of the 21 dimer pairs along the pathline of maximum soot along the 
wings (top), and the centerline (bottom), for the four models. Along the wings and centerline, the dimer pairs that contribute the most to nucleation are pair 1 (two 10 carbon PAHs, or A2-A2) and 7 (two 12 carbon PAHs, or A2R5-A2R5), which are the smaller dimer pairs. Along the centerline, pair 1 is dominant for the greatest range of carbonization rates, while pair 7 is dominant for the greatest range on the wings. The improved predictions for aggregate number density along the centerline in Section 4.1 .2 can be attributed to including smaller dimer pairs in the nucleation process. As the carbonization rate is decreased, there is a trend of larger dimer pairs having a greater contribution to the nucleation process. Along the wings, Model C08 shows significant contributions from dimer pair 11 (10-20, or A2R5BAPYR) and dimer pair 21 (20-20, or BAPYR-BAPYR). However, Model C08 has poor agreement with experimental soot primary particle diameters on the centerline, which can be seen in the supplemental material.

Fig. 4 displays the total path integrated contribution to PAH condensation for each of the $6 \mathrm{PAH}$ size groups along the pathline of maximum soot along the wings (top), and the centerline (bottom) for the four models. Along the wings and centerline, both the relatively small and large PAHs play a significant role in the condensation process with the highest contributions from PAHs with 12 carbons (A2R5) and those with 18 carbons (BGHIF). Similar to nucleation, as the carbonization rate is reduced, larger PAHs have greater contributions to the condensation process. It should be noted that while not shown, the trends in the species contributing to nucleation and condensation are not the same if efficiencies are utilized instead of reversibility along with a carbonization rate. 


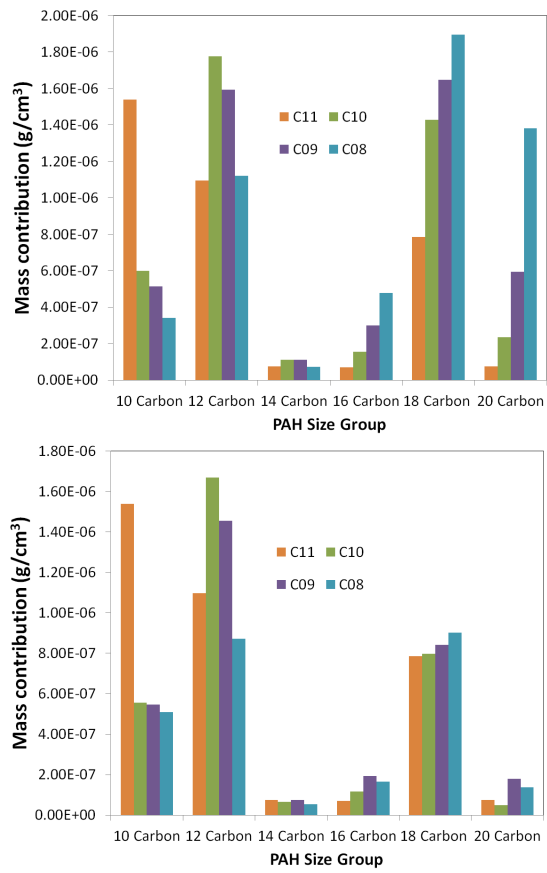

Figure 4: Total path integrated contribution to $\mathrm{PAH}$ condensation for each of the $6 \mathrm{PAH}$ size groups for four models along the pathline of maximum soot along the wings (top), and the centerline (bottom) for a co-flow ethylene-air diffusion flame.

In summary, the results display that smaller PAHs are dominant for the nucleation process, while a broad range of PAHs are important for the condensation process. It should be noted that even over four orders of magnitude, the trends in which species contribute are largely insensitive to the carbonization rate. Thus, the results are not purely a function of the chosen carbonization rate. To understand this result, an additional calculation is performed with Model $\mathrm{C} 10$ with all condensation rates equal to zero, or in other words, PAHs can only undergo a nucleation process. Fig. 5 displays the total path integrated contribution to soot nucleation for each of the 21 dimer pairs, for Model C10 without condensation, along the pathline 
of maximum soot along the wings (top), and the centerline (bottom). In the absence of condensation, the importance of larger dimer pairs to nucleation is greater than when condensation is permitted. On the wings, dimer pair 19 is dominant (18-20, or BAPYR-BGHIF), which is the third largest dimer pair considered. While on the centerline a smaller dimer pair, pair 7 (A2R5A2R5), is dominant, significant contributions are seen from dimer pair 19 as well.

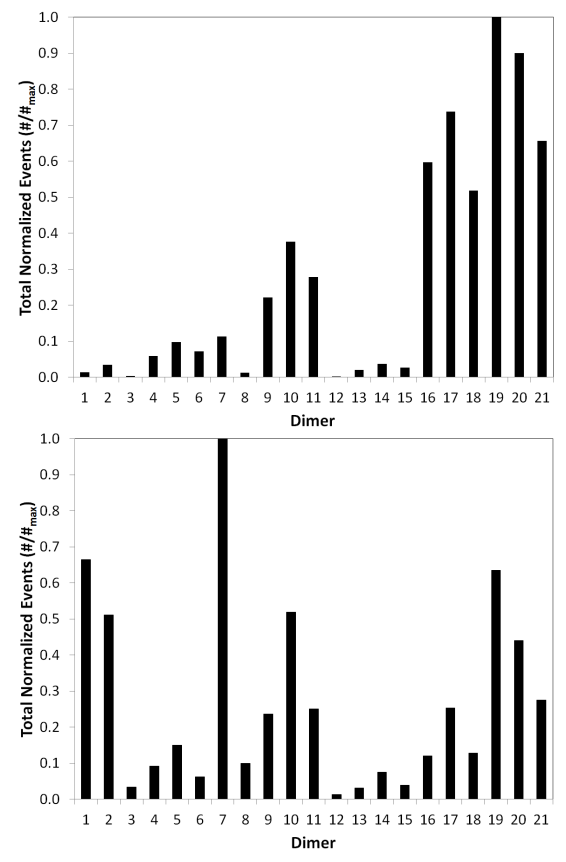

Figure 5: Total path integrated contribution to soot nucleation for each of the 21 dimer pairs, for Model C10 without condensation, along the pathline of maximum soot along the wings (top), and the centerline (bottom) for a co-flow ethylene-air diffusion flame.

From these results in Fig. 5, the reason for the observed importance of smaller dimer pairs in Fig. 3 is due to the presence of condensation. In the RPC model, the condensation process is less reversible than the nucleation 
process due to two factors: increased binding energies and lower frequencies of the vibration modes created [12]. Even though the forward rate of nucleation may be stronger than that of condensation due to increased collision probability, the thermodynamics (and hence reverse rate) heavily favors condensation. While there is uncertainty in the frequency of the vibrational modes, a lower frequency is consistent with literature [5, 6]. Additionally, the enhanced RPC model demonstrates good agreement for primary particle diameter, displaying that the ratio of nucleation to condensation strength is reasonable, as primary particle size predictions are very sensitive to the relative strengths of these two processes [30]. Thus, once a dimer, or cluster, is formed from an initial pool of PAHs, it is more probable that remaining free PAHs will condense on the exist cluster, rather than form a new cluster or dimer. Since smaller PAHs are formed prior to larger PAHs on a given streamline in diffusion flames (although there is some evidence against the generality of this statement [43]), the initial clusters are formed from these smaller PAHs. Subsequently, any larger PAHs forming in the gas phase preferentially condense rather than dimerize, leading to the low contribution of larger PAHs to the nucleation process. Due to uncertainties in the gas-phase mechanism and the employed soot model, it cannot be stated exactly how small PAHs may be and still be important to the nucleation process; however, the trend of relative smaller PAHs contributing to nucleation can be stated with confidence. If a different gas-phase mechanism is employed, the results may indicate that different dimer pairs are contribute the most to nucleation, thus the conclusions of this study are limited to the general trends rather than identifying specific dimer pairs. 
The result of smaller PAHs being important to the nucleation process is consistent with the literature. Teini et al [10] utilized HRTEM to investigate the sizes of PAHs within nascent soot particles by analyzing fringe lengths. It was shown that the average size of PAHs were those containing 20 carbons; however, the mode of the distribution were PAHs with 12 carbons (A2R5). Apicella et al [44] utilized HRTEM to investigate PAHs with soot particles. Based on the diameters of the basic structure units (BSU) from nascent soot (low axial heights) reported in the supplemental material, the results indicate nascent soot primarily contained PAHs with 12 carbons as well. While the results of Adkins and Miller [45] for optical band gap (OBG) measurements suggest that PAHs containing 14 rings, or 42 carbons, are important to the nucleation process, the work of Commodo et al $[46,47]$ suggests that the resulting OBG measurements are a stronger function of the number of PAHs within BSU stacks, rather than the size of the individual PAHs within soot particles. While theoretical studies suggest that small PAH dimerization is not thermodynamically favored, unfavored does not mean impossible. Also, these theoretical studies neglect the possibility of chemical bonding. The enhanced RPC model utilizes parameters derived by these theoretical studies and includes the effect of chemical bonding, thus the conclusions of this study are congruent with previous theoretical studies [2-9].

\section{Conclusions}

The original reversible PAH clustering (RPC) model is enhanced to include 21 dimerization events and tracking of internal PAHs within soot particles. The enhanced RPC model shows improved agreement with experimen- 
tal measurements for an ethylene-air coflow diffusion flame. Even though there is uncertainty in the carbonization rate, when investigating rates that span four orders of magnitude, the conclusions of this work are consistent. Smaller PAHs contribute the most to nucleation processes due to condensation being less reversible than nucleation and the step-wise nature of PAH growth in diffusion flames, while small and large PAHs are important for the condensation process. While the exact contribution from each PAH size group will depend on the predictions of gas phase PAHs from the chemical mechanism, the general trends observed in this study are not expected to change as the trends can be explained based on the fundamental knowledge of the nucleation and condensation processes. Future work should focus on developing a fundamental model for the carbonization process. Other work should focus on investigation the role of additional chemical growth species beyond acetylene as well.

\section{Acknowledgments}

The authors acknowledge NSERC of Canada for additional financial support. Computations were performed on the Ryerson University Sandy Bridge computing cluster and the GPC supercomputer at the SciNet HPC Consor-

tium. SciNet is funded by: the Canada Foundation for Innovation under the auspices of Compute Canada; the Government of Ontario; Ontario Research Fund - Research Excellence; and the University of Toronto. 


\section{References}

[1] H. Sabbah, L. Biennier, S. J. Klippenstein, I. R. Sims, B. R. Rowe, Journal of Physical Chemistry Letters 1 (2010) 2962-2967.

[2] H. Wang, Proceedings of the Combustion Institute 33 (2011) 41-67.

[3] J. D. Herdman, J. H. Miller, Journal of Physical Chemistry A 112 (2008) $6249-6256$.

[4] T. S. Totton, A. J. Misquitta, M. Kraft, Physical Chemistry Chemical Physics 14 (2012) 4081-4094.

[5] M. Rapacioli, F. Calvo, C. Joblin, P. Parneix, F. Spiegelman, Journal of Physical Chemistry A 111 (2007) 2999-3009.

[6] M. Rapacioli, F. Calvo, F. Spiegelman, C. Joblin, D. J. Wales, Journal of Physical Chemistry A 109 (2005) 2487-2497.

[7] P. Elvati, A. Violi, Proceedings of the Combustion Institute 34 (2013) $1837-1843$.

[8] S. Chung, A. Violi, Proceedings of the Combustion Institute 33 (2011) 693-700.

[9] J. S. Lowe, J. Y. W. Lai, P. Elvati, A. Violi, Proceedings of the Combustion Institute 35 (2015) 1827-1832.

[10] P. D. Teini, D. M. A. Karwat, A. Atreya, Combustion and Flame 158 (2011) 2045-2055. 
[11] R. A. Dobbins, R. A. Fletcher, H. . Chang, Combustion and Flame 115 (1998) 285-298.

[12] N. A. Eaves, S. B. Dworkin, M. J. Thomson, Proceedings of the Combustion Institute 35 (2015) 1787-1794.

[13] R. J. Santoro, H. G. Semerjian, R. A. Dobbins, Combustion and Flame 51 (1983) 203-218.

[14] R. J. Santoro, T. T. Yeh, J. J. Horvath, H. Semerjian, Combustion Science and Technology 53 (1987) 89-115.

[15] C. M. Megaridis, R. A. Dobbins, Combustion Science and Technology 66 (1989) 1-16.

[16] R. Puri, T. F. Richardson, R. J. Santoro, R. A. Dobbins, Combustion and Flame 92 (1993) 320-333.

[17] N. A. Eaves, Q. Zhang, F. Liu, H. Guo, S. Dworkin, M. Thomson, Computer Physics Communications (2016).

[18] S. V. Patankar, Numerical Heat Transfer and Fluid Flow, Hemisphere, New York, 1980.

[19] N. A. Eaves, M. J. Thomson, S. B. Dworkin, Combustion Theory and Modelling 185 (2013) 1799-1819.

[20] N. A. Slavinskaya, P. Frank, Combustion and Flame 156 (2009) 17051722 . 
[21] S. B. Dworkin, Q. Zhang, M. J. Thomson, N. A. Slavinskaya, U. Riedel, Combustion and Flame 158 (2011) 1682-1695.

[22] N. A. Slavinskaya, U. Riedel, S. B. Dworkin, M. J. Thomson, Combustion and Flame 159 (2012) 979-995.

[23] Q. Zhang, H. Guo, F. Liu, G. J. Smallwood, M. J. Thomson, Proceedings of the Combustion Institute 32 (2009) 761-768.

[24] N. A. Eaves, A. Veshkini, C. Riese, Q. Zhang, S. B. Dworkin, M. J. Thomson, Combustion and Flame 159 (2012) 3179-3190.

[25] M. Saffaripour, Q. Kholghy, M. Zhang, S. B. Dworkin, M. J. Thomson, Proceedings of the Combustion Institute 32 (2013) 1057-1065.

[26] V. Chernov, Q. Zhang, M. J. Thomson, S. B. Dworkin, Combustion and Flame 159 (2012) 2789-2798.

[27] V. Chernov, M. J. Thomson, S. B. Dworkin, N. A. Slavinskaya, U. Riedel, Combustion and Flame 161 (2014) 592-601.

[28] A. Veshkini, S. B. Dworkin, M. J. Thomson, Combustion and Flame 161 (2015) 3191-3200.

[29] A. Khosousi, S. B. Dworkin, Proceedings of the Combustion Institute (2014). Http://dx.doi.org/10.1016/j.proci.2014.05.152.

[30] M. Saffaripour, A. Veshkini, M. Kholghy, M. J. Thomson, Combustion and Flame 161 (2014) 848-863. 
[31] Q. Zhang, M. J. Thomson, H. Guo, F. Liu, G. J. Smallwood, Combustion Science and Technology 182 (2010) 491-504.

[32] Q. Zhang, H. Guo, F. Liu, G. J. Smallwood, M. J. Thomson, Combustion Theory and Modelling 12 (2008) 621-641.

[33] F. Liu, S. B. Dworkin, M. J. Thomson, G. J. Smallwood, Combustion Science and Technology 184 (2012) 966-979.

[34] S. H. Park, S. N. Rogak, W. K. Bushe, J. Z. Wen, M. J. Thomson, Combustion Theory and Modelling 9 (2005) 499-513.

[35] S. H. Park, S. N. Rogak, Journal of Aerosol Science 35 (2004) 1385-1404.

[36] R. A. Dobbins, G. J. Govatzidakis, W. Lu, A. F. Schwartzman, R. A. Fletcher, Combustion Science and Technology 121 (1996) 103-121.

[37] G. Basile, A. Rolando, A. D’Alessio, A. D’Anna, P. Minutolo, Proceedings of the Combustion Institute 29 (2002) 2391-2397.

[38] D. Chen, T. S. Totton, J. W. J. Akroyd, S. Mosbach, M. Kraft, Carbon 67 (2014) 79-91.

[39] Ü. Ö. Köylü, C. S. Mcenally, D. E. Rosner, L. D. Pfefferle, Combustion and Flame 110 (1997) 494-507.

[40] C. S. McEnally, Ü. Ö. Köylü, L. D. Pfefferle, D. E. Rosner, Combustion and Flame 109 (1997) 701-720.

[41] J. Y. W. Lai, P. Elvati, A. Violi, Physical Chemistry Chemical Physics 16 (2014) 7969-7979. 
[42] B. Shukla, M. Koshi, Combustion and Flame 159 (2012) 3589-3596.

[43] K. O. Johansson, J. Y. W. Lai, S. A. Skeen, D. M. Popolan-Vaida, K. R. Wilson, N. Hansen, A. Violi, H. A. Michelsen, Proceedings of the Combustion Institute 35 (2015) 1819-1826.

[44] B. Apicella, P. Pr, M. Alf, A. Ciajolo, V. Gargiulo, C. Russo, A. Tregrossi, D. Deldique, J. N. Rouzaud, Proceedings of the Combustion Institute 35 (2015) 1895-1902.

[45] E. M. Adkins, J. H. Miller, Physical Chemistry Chemical Physics 17 (2015) 2686-2695.

[46] M. Commodo, G. Tessitore, G. De Falco, A. Bruno, P. Minutolo, A. D'Anna, Proceedings of the Combustion Institute 35 (2015) 17951802.

[47] M. Commodo, G. De Falco, A. Bruno, C. Borriello, P. Minutolo, A. D'Anna, Combustion and Flame (2015). Article in Press. 
One supplemental materials document accompanies this manuscript.

Title: Model Details and Soot Morphological Parameter Validation

Caption: Details of the enhanced RPC model along with validation of the enhanced model against experimental data for soot morphological parameters for the Santoro ethylene-air diffusion flame. 